\title{
HELSON SETS WHICH DISOBEY SPECTRAL SYNTHESIS
}

\section{SADAHIRO SAEKI}

ABSTRACT. In this paper it is shown that every nondiscrete LCA group contains a compact independent $\mathrm{Hel}$ son set which disobeys spectral synthesis.

In [3] T. W. Körner has constructed an independent compact $H_{1}$-set of type $M$. This result solves negatively the long-standing problem whether or not every Helson set obeys spectral synthesis. Körner's construction of such a set is, however, very complicated, and R. Kaufman [2] has simplified it (see also [6]. In this paper, we modify Kaufman's method to prove that every nondiscrete metrizable LCA group contains an independent compact $\mathrm{Hel}$ son set of type $M$. Consequently it is shown that every nondiscrete LCA group contains a Helson set which disobeys spectral synthesis.

Let $G$ be a LCA group with dual $\hat{G}$. We denote by $A(G)$ and $P M(G)$ the Fourier algebra on $G$ and the conjugate space of $A(G)$, respectively. Each element of $P M(G)$ is called a pseudo-measure on $G$. For $f \in A(G)$ and $P \in P M(G)$, we define

$$
\langle f, P\rangle=(\hat{f} * \hat{P})(1)=\int_{\hat{G}} \hat{f}(\chi) \hat{P}\left(\chi^{-1}\right) d \chi,
$$

where $\hat{f}$ and $\hat{P}$ denote the functions in $L^{1}(\hat{G})$ and in $L^{\infty}(\hat{G})$ whose (inverse) Fourier transforms are $f$ and $P$, respectively. If $\mu \in M(\hat{G})$ and $\breve{\mu}(x)=$ $\int_{\widehat{G}} \chi(x) d \mu(\chi)(x \in G)$, then we denote by $\breve{\mu} P$ the pseudo-measure on $G$ defined by the requirement $(\breve{\mu} P)^{\wedge}=\mu * \hat{P}$. It is well known that if $P \in P M(G)$ has compact support, then $\hat{P}$ can be chosen from the space $C(G)$; we will always do this. For such a $P,\langle\breve{\mu}, P\rangle=(\mu * \hat{P})(1)(\mu \in M(\hat{G}))$ is well defined, and we have $\langle\chi, \gamma P\rangle=\hat{P}\left(\chi^{-1} \gamma^{-1}\right)(\chi, \gamma \in \hat{G})$. A p seudo-function on $G$ is any pseudo-measure whose Fourier transform is a continuous function on $\hat{G}$ which vanishes at infinity. The space of all pseudo-functions is denoted by $P F(G)$.

Received by the editors July 25, 1973.

AMS (MOS) subject classifications (1970). Primary 43A45; Secondary 43A20.

Key words and phrases. LCA group, Helson set, set of type $M, H_{1}$-set, $K_{q^{-s e t}}$, Fourier algebra, spectral synthesis, pseudo-function. 
Let now $E$ be a closed set in $G$, and $q=q(G)$ the supremum of the natural numbers $n$ such that every neighborhood of $0_{G}$ contains an element with order $\geq n$. $E$ is called an $M$-set (or a set of type $M$ ) if $P F(E) \neq 0$ (that is, if $E$ carries a nonzero pseudo-function); strongly independent if $E$ is independent in the usual sense and $\operatorname{ord}(e)=q$ for all $e \in E$; and an $H_{1}$-set if $\|\mu\|_{M}=\|\mu\|_{P M}$ for all $\mu \in M(E)$. In the case $q<\infty, E$ is called a $K_{q}$-set if every function $f \in C(E)$ with $f^{q}=1$ is the restriction of a character in $\hat{G}$; and a weak $K_{q}$-set if to each $\mu \in M^{+}(E)$ and $\epsilon>0$ there corresponds a $K_{q}$-set $K \subset E$ such that $\mu(E \backslash K)<\epsilon$.

Our main result now follows (cf. [3, p. 105]).

Theorem 1. Every nondiscrete LCA group contains a strongly independent compact set which is either an. $H_{1}$-set or a weak $K_{q}$-set but disobeys spectral synthesis. If, in addition, the group under consideration is metrizable, such a set can be chosen as a set of type M.

The proof becomes clear after some lemmas and theorems are established.

Lemma 1. To each $0<\epsilon<1$ there corresponds a natural number $N_{\epsilon}$ with the following property: for any neighborhood $V$ of 0 of a compact abelian group $H$ and any natural number $N \geq N_{\epsilon}$, there exists an $F \in A\left(H^{N}\right)$ such that

(i) $\operatorname{supp} F \subset V_{\epsilon}^{N}$, and

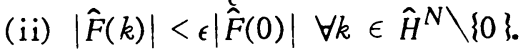

Here $V_{\epsilon}^{N}$ is the set of all $\left(x_{j}\right)_{1}^{N} \in H^{N}$ such that $x_{j} \in V$ for at least $(1-\epsilon) N$ indices $j=1,2, \cdots, N$, and $\hat{H}^{N}$ denotes the additively written dual of $H^{N}$.

The proof is essentially identical with that of [2, Lemma 2], so we omit it.

Lemma 2. Let $G$ be a LCA I-group, $\hat{K}$ a compact subset of $\hat{G}$, and $M$, $N \in \mathbf{N}$ (the natural numbers). The ${ }_{k}$ there exist $N$ characters $\chi_{1}, \cdots, \chi_{N}$ $\epsilon \hat{G}$ such that the sets $\chi_{1}^{k_{1}} \cdots \chi_{N}^{k^{N}} \hat{K}\left(k_{j} \in\{0, \pm 1, \cdots, \pm M\}, 1 \leq j \leq N\right)$ are pairwise disjoint.

Proof. By the structure theorem $[1,(9.8)], G$ contains an open subgroup $G_{0}$ which is topologically isomorphic to $R^{a} \times H$ for some $a \in\{0,1,2, \cdots\}$ and some compact abelian group $H$. Since there exists a continuous homomorphism of $\hat{G}$ onto $\hat{G}_{0}$, we may assume that $G=G_{0}=R^{a} \times H$.

Suppose that there exists a $\gamma \in \hat{G}$ such that 


$$
\lim _{n} \gamma^{n}=\infty \text {. }
$$

Take $p \in N$ so large that $n>p \quad \gamma^{n} \notin \hat{K}^{-1} \hat{K}$, and choose $N$ natural numbers $q_{1}, \cdots, q_{N}$ so that $\left|k_{1} q_{1}+\cdots+k_{N} q_{N}\right|>p$ for all choices of $k_{j} \epsilon$ $\{0, \pm 1, \pm 2, \cdots, \pm 2 M\}, 1 \leq j \leq N$, such that $\left(k_{1}, \cdots, k_{N}\right) \neq(0, \cdots, 0)$. Then the elements $\chi_{j}=\gamma^{\bar{q}}, 1 \leq j \leq N$, have the required property.

Assume now that no $\gamma \in \hat{G}$ satisfies (1). Then $a=0, G=H$ is compact and $\hat{G}$ is a torsion group. Therefore every finitely-generated subgroup of $\hat{G}$ is finite. But $\hat{G}$ is not of bounded order because $G$ is an I-group (see $[4,2.5 .4])$. Thus, setting $\chi_{0}=1$, we can find $\chi_{1}, \cdots, \chi_{N} \in \hat{G}$ so that

$$
\operatorname{ord}\left(\chi_{j}\right)>2 M \cdot \operatorname{Card}\left[G_{p}\left(\hat{K} \cup\left\{\chi_{0}, \cdots, \chi_{j-1}\right\}\right)\right]
$$

for all $j=1,2, \cdots, N$. As is easily seen, the $\chi_{j}, 1 \leq j \leq N$, have the required property.

Lemma 3. Let $G$ be a LCA I-group, and $\tau$ a pseudo-function on $G$ whose support $E$ is compact. Let also $0<\epsilon<1$, and $g$ any function in $C(E)$ whose range is a finite subset of $T=\{z:|z|=1\}$. Then there exist $\tau^{\prime} \in P F(G)$ and $\chi_{j} \in \hat{G}, 1 \leq j \leq N$, such that
(a) $\left\|\tau^{\prime}-\tau\right\|_{P M}<\epsilon$,
(b) supp $\tau^{\prime} \subset$ supp $\tau$,
(c) $\left\|g-(1 / N) \Sigma_{j=1}^{N} \chi_{j}\right\|_{C\left(\operatorname{supp} \tau^{\prime}\right)}<\epsilon$.

Proof. We may assume that $g$ is defined and continuous on some compact neighborhood $U$ of $E$, and that $g(U)$ is a finite subset of $T$. Thus

$$
C=\sup \left\{\left\|g^{k}\right\|_{A(U)}: k \in \mathbf{Z}\right\}<\infty \text {, }
$$

where

$$
\left\|g^{k}\right\|_{A(U)}=\inf \left\{\|f\|_{A(G)}: f \in A(G), f=g^{k} \text { on } U\right\} .
$$

Put $V=\{z \in T:|z-1|<\epsilon\}$, so that

$$
V_{\epsilon}^{N}=\left\{\left(z_{j}\right)_{1}^{N} \in T^{N}: \operatorname{Card}\left\{j:\left|z_{j}-1\right|<\epsilon\right\} \geq(1-\epsilon) N\right\}
$$

for all $N \in \mathbf{N}$. We apply Lemma 1 to find an $N \in \mathbf{N}$ and an $F \in A\left(T^{N}\right)$ such that

$$
\begin{gathered}
\operatorname{supp} F \subset V_{\epsilon}^{N}, \\
\hat{F}(0)=1 \text { and }|\hat{F}(k)|<\epsilon \quad-k \in \mathbf{Z}^{N} \backslash\{0\} .
\end{gathered}
$$


Choose a finite set $L \subset \mathbf{Z}^{N}$ so that

$$
\sum_{k \notin L}|\hat{F}(k)| \cdot C\|\tau\|_{P M}<\epsilon,
$$

and put

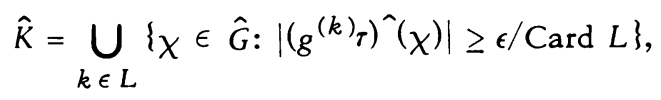

where $(k)=\Sigma_{j} k_{j}$ for $k=\left(k_{1}, \cdots, k_{N}\right) \in \mathbf{Z}^{N}$. Since $g^{(k)} \tau \in P F(G)$ and $L$ is finite, $\hat{K}$ is compact. It follows from Lemma 2 that there are $N$ characters $\chi_{1}, \cdots, \chi_{N} \in \hat{G}$ such that the sets

$$
\chi_{1}^{-k_{1}} \cdots \chi_{N}^{-k} \hat{K} \hat{K} \quad(k \in L)
$$

are pairwise disjoint. Note that the series

$$
\sum_{k \in \mathbf{Z}^{N}} \hat{F}(k) g^{(k)} \bar{\chi}_{1}^{k} \cdots \bar{\chi}_{N}^{k}
$$

converges to $F\left(g \bar{X}_{1}, \cdots, g \bar{X}_{N}\right) \in A(U)$ in the norm of $A(U)$ by (1). Setting

$$
\tau^{\prime}=F\left(g \bar{\chi}_{1}, \cdots, g \bar{\chi}_{N}\right) \tau=\sum_{k \in Z^{k}} \hat{F}(k) g^{(k)} \bar{\chi}_{1}^{k} \cdots \bar{\chi}_{N}^{k} r,
$$

we claim that $\tau^{\prime}$ and $\chi_{1}, \cdots, \chi_{N}$ have the required properties if $\epsilon$ is replaced by $\epsilon /\left(C\|\tau\|_{P M}+3\right)$.

To prove part (a), put

$$
\nu=\sum_{0 \neq k \in L} \hat{F}(k) g^{(k)} \bar{\chi}_{1}^{k} \cdots \bar{\chi}_{N}^{k} N^{r}
$$

Then we have

$$
\begin{aligned}
|\hat{\nu}(\chi)| & \leq \sum_{0 \neq k \in L}|\hat{F}(k)| \cdot\left|\left(g^{(k)} \tau\right)^{\wedge}\left(\chi_{1}^{k_{1}} \cdots \chi_{N}^{k_{N}} \chi\right)\right| \\
& \leq \epsilon \sum_{k \in L}\left|\left(g^{(k)} \tau\right)^{\wedge}\left(\chi_{1}^{k_{1}} \cdots \chi_{N}^{k} \chi\right)\right|
\end{aligned}
$$

for all $\chi \in \hat{G}$ by (4). If one of the summands in the last sum is $\geq \epsilon /$ Card $L$, then the other summands are $<\epsilon /$ Card $L$ by (6) and (7). Therefore, $|\hat{\nu}(\chi)| \leq$ $\epsilon\left(C\|\tau\|_{P M}+1\right)$ by (1); hence $\|\nu\|_{P M} \leq \epsilon\left(C\|t\|_{P M}+1\right)$. It follows from (8), (4), and (5) that 


$$
\begin{aligned}
\left\|\tau^{\prime}-\tau\right\|_{P M} & =\left\|\sum_{k \neq>0} \hat{F}(k) g^{(k)} \bar{\chi}_{1}^{k} \cdots \bar{\chi}_{N}^{k} N\right\|_{P M} \\
& \leq\|\nu\|_{P M}+\sum_{k \notin L}|\hat{F}(k)| \cdot\left\|g^{(k)} \tau\right\|_{P M} \\
& <\epsilon\left(C\|r\|_{P M}+1\right)+\epsilon=\epsilon\left(C\|\tau\|_{P M}+2\right) .
\end{aligned}
$$

Part (b) is trivial from (8).

To verify (c), note that supp $\tau^{\prime}$ is contained in the closed set $\{x \in E$ : Card $\left.\left\{j:\left|g(x)-\chi_{j}(x)\right| \leq \epsilon\right\} \geq(1-\epsilon) N\right\}$, which is easily seen from (2), (3), and (8). Thus $x \in \operatorname{supp} \tau^{\prime}$ implies

$$
\left|g(x)-N^{-1} \sum_{j=1}^{N} \chi_{j}(x)\right| \leq N^{-1} \sum_{j=1}^{N}\left|g(x)-\chi_{j}(x)\right| \leq \epsilon+N^{-1} \cdot 2 \epsilon N=3 \epsilon .
$$

This completes the proof.

Theorem 2. Let $G$ be a LCA I-group and $\tau$ a pseudo-function on $G$ whose support $E$ is totally disconnected and metrizable. Then for each $\epsilon>$ 0 there exists a $\nu \in P F(E)$, with $\|\nu-\tau\|_{P M}<\epsilon$, whose support is a strongly independent $H_{1}$-set.

Proof. Since $\hat{\tau}$ is uniformly continuous, we may assume that $E$ is compact. Since $E$ is metrizable, $C(E)$ is separable, and so $\{f \in C(E):|f|=1\}$ contains a countable dense set $\left\{g_{n}\right\}$. We may assume $\operatorname{Card}\left[g_{n}(E)\right]<\infty$ for all $n$, because $E$ is totally disconnected.

Let $\epsilon>0$ be given, and set $\tau_{0}=\tau$. Suppose that $\tau_{0}, \cdots, \tau_{n-1} \in P F(E)$ are constructed for some natural number $n$. We apply Lemma 3 to find a ${ }_{n}$ $\epsilon P F(E)$ which satisfies (a), (b), and (c) in Lemma 3 with $r, \epsilon, g$ replaced by $\tau_{n-1}, \epsilon / 2^{n}, g_{n}$, respectively. The sequence $\left\{\tau_{n}\right\}_{1}^{\infty}$ obtained in this way converges to some $\nu \in P F(E)$ and we have

$$
\|\nu-\tau\|_{P M} \leq \sum_{n=1}^{\infty}\left\|\tau_{n}-\tau_{n-1}\right\|_{P M}<\epsilon .
$$

Clearly supp $\nu \subset \bigcap_{n}$ supp $\tau_{n^{*}}$. Thus, for each $n \in \mathbf{N}$, there are finitely many characters $\chi_{1}, \cdots, \chi_{N} \in \hat{G}$ such that

$$
\left\|g_{n}-\frac{1}{N} \sum_{j=1}^{N} \chi_{j}\right\|_{C(\operatorname{supp} \nu)}<\frac{\epsilon}{2^{n}} .
$$


This implies that supp $\nu$ is an $H_{1}$-set which is independent over $Z$.

Lemma $2^{\prime}$. Let $G$ be a nondiscrete LCA group with $q=q(G)<\infty, E \subset$ $G$ and $\hat{K} \subset \hat{G}$ compact, and $N \in \mathbf{N}$. Then there are $N$ characters $\chi_{1}, \cdots$, $\chi_{N} \in \hat{G}$ with the following two properties:

(i) The sets $\chi_{1}^{k_{1}} \cdots \chi_{N}{ }^{N} \hat{K}\left(k_{j} \in\{0,1, \cdots, q-1\}, 1 \leq j \leq N\right)$ are pairwise disjoint;

(ii) $\chi_{j}^{q}=1$ on some neighborhood of $E(1 \leq j \leq N)$.

Proof. We may assume that $G$ is compactly generated. Thus $G=R^{a} \times$ $Z^{b} \times H$ for some nonnegative integers $a$ and $b$ and some compact abelian group $H$ (see $[1,(9.8)]$ ). Since $q<\infty, a=0$ and $H$ is of bounded order. Let $\hat{K}_{0}$ be the natural projection of $\hat{K}$ into $\hat{H}$. Since $H$ is a compact group with $q(H)=q(G)=q<\infty$, we can find $N_{k} N$ characters $\gamma_{1}, \cdots, \gamma_{N} \in \hat{H}$ with order $q$ so that the sets $\gamma_{1}^{k} \cdots \gamma_{N}^{k} \hat{K}_{0}\left(k_{j} \in\{0,1, \cdots, q-1\}, 1 \leq j \leq N\right)$ are pairwise disjoint. (Note that $\hat{H}$ is a weak direct product of finite cyclic groups [4, B8].) Setting $\chi_{j}=1 \otimes \gamma_{j} \in T^{b} \times \hat{H}=\hat{G}$, we see that the elements $\chi_{1}, \cdots, \chi_{N}$ have the required property.

Lemma $3^{\prime}$. Let $G$ be a nondiscrete LCA group with $q<\infty$, and $\tau$ a pseudo-function on $G$ with compact support $E$. Let also $0<\epsilon<1$, and $g$ any function in $C(E)$ with $g^{q}=1$. Then there exists a $\tau^{\prime} \in P F(G)$ such that

(a) $\left\|\tau^{\prime}-\tau\right\|_{P M}<\epsilon$;

(b) $\operatorname{supp} \tau^{\prime} \subset \operatorname{supp} \tau$;

(c) $\left\|g-(1 / N) \Sigma_{j=1}^{N} \chi_{j}\right\|_{C\left(\text { supp } \tau^{\prime}\right)}<\epsilon$

for some characters $\chi_{1}, \cdots, \chi_{N} \in \hat{G}$ with $\chi_{j}^{q}=1$ on $E$.

The proof is very similar to that of Lemma 3. The needed modifications are as follows. Replace $T$ and $V$ by $T_{q}=\left\{z \in T: z^{q}=1\right\}$ and $\{1\} \subset T_{q}$, respectively; realize the dual of $T_{q}^{N}$ as $\{0,1, \cdots, q-1\}^{N}$ in the usual way; put $L=\{0,1, \cdots, q-1\}^{N}$; and use Lemma $2^{\prime}$ instead of Lemma 2 .

The following theorem can be proved by applying Lemma $3^{\prime}$ just as Theorem 2 was proved. We omit the proof.

Theorem 3. Let $G$ be a nondiscrete LCA group with $q=q(G)<\infty$, and $\tau$ a pseudo-function on $G$ whose support $E$ is metrizable. Then, given $\epsilon>0$, we can find a pseudo-function $\nu \in P F(E)$, with $\|\nu-\tau\|_{P M}<\epsilon$, whose support $K$ has the following property: to each $\delta>0$ and $g \in C(K)$ with $g^{q}=1$, there correspond finitely many characters $\chi_{1}, \cdots, \chi_{N} \in \hat{G}$ such that $\chi_{j}^{q}=1$ on $K(1 \leq j \leq N)$ and $\left\|g-(1 / N) \sum_{j=1}^{N} \chi_{j}\right\|_{C(K)}<\delta$. 
It is now a routine matter to derive Theorem 1 from Theorems 2 and 3 because every LCA group $G$ contains a closed metrizable group $H$ with $q(H)=q(G)$, and every Helson set of type $M$ disobeys spectral synthesis (cf. [4, 5.6.10]).

Remarks. (a) For a characterization of Hel son sets of (spectral) synthesis, we refer to [5].

(b) The totally disconnectedness assumption on $E$ in Theorem 2 is unnecessary. Let $E$ be a closed metrizable subset of $G$, and $\tau \in P F(E)$. Then there exists a sequence $\left\{\tau_{n}\right\}$ in $P F(E)$ such that supp $\tau_{n}$ is totally disconnected and $\left\|\tau_{n}-\tau\right\|_{P M}=O(1)$. We omit the proof.

\section{REFERENCES}

1. E. Hewitt and K. A. Ross, Abstract harmonic analysis. Vol. I: Structure of topological groups. Integration theory, group representations, Die Grundlehren der math. Wissenschaften, Band 115, Academic Press, New York; Springer-Verlag, Berlin, 1963. MR 28 \# 158.

2. R. Kaufman, M-sets and distributions, Astérisque Math. France 5 (1973), $225-230$.

3. T. W. Kömer, A pseudo-function on a Helson set, Astérisque Math. France 5 ( 1973), 3-224.

4. W. Rudin, Fourier analysis on groups, Interscience Tracts in Pure and Appl. Math., no. 12, Interscience, New York, 1962. MR 27 \# 2808.

5. S. Saeki, A characterization of SH-sets, Proc. Amer. Math. Soc. 30 (1971), 497-503. MR $44 \# 731$.

6. T. W. Körner, A pseudo-function on a Helson set. II, Astérisque Math. France 5 (1973), 231-239.

DEPARTMENT OF MATHEMATICS, KANSAS STATE UNIVERSITY, MANHATTAN, KAN SAS 66506

Current address: Department of Mathematics, Tokyo Metropolitan University, Setagaya, Tokyo, Japan 